\title{
Identification of a new caspase homologue: caspase-14
}

\author{
Marc Van de Craen ${ }^{1}$, Geert Van Loo ${ }^{1}$, Stefan Pype ${ }^{2}$, \\ Wim Van Criekinge ${ }^{1}$, Ilse Van den brande ${ }^{1}$, \\ Francis Molemans ${ }^{1}$, Walter Fiers ${ }^{1}$, Wim Declercq ${ }^{1}$ and \\ Peter Vandenabeele ${ }^{1,3}$ \\ ${ }^{1}$ Department of Molecular Biology, Flanders Interuniversity Institute for \\ Biotechnology and University of Ghent, Ghent, Belgium \\ 2 Department of Cell Growth, Differentiation and Development, Flanders \\ Interuniversity Institute for Biotechnology and Catholic University of Leuven, \\ Leuven, Belgium \\ ${ }^{3}$ corresponding author: P. Vandenabeele, Department of Molecular Biology, \\ K.L. Ledeganckstraat 35, B-9000 Ghent, Belgium. \\ tel: 32-9-264-51-31; fax: 32-9-264-53-48; \\ e-mail: peter.vandenabeele@Imb.rug.ac.be. \\ The first two authors contributed equally to this work.
}

Received 2.2.98; revised 16.7.98; accepted 3.8.98.

Edited by G. Melino

\begin{abstract}
Caspases are cysteinyl aspartate-specific proteinases, many of which play a central role in apoptosis. Here, we report the identification of a new murine caspase homologue, viz. caspase-14. It is most related to human/murine caspase-2 and human caspase-9, possesses all the typical amino acid residues of the caspases involved in catalysis, including the QACRG box, and contains no or only a very short prodomain. Murine caspase- 14 shows $83 \%$ similarity to human caspase14. Human caspase-14 is assigned to chromosome 19p13.1. Northern blot analysis revealed that mRNA expression of caspase- 14 is undetectable in all mouse adult tissues examined except for skin, while it is abundantly expressed in mouse embryos. In contrast to many other caspase family members, murine caspase- 14 is not cleaved by granzyme $B$, caspase-1, caspase-2, caspase-3, caspase- 6 , caspase- 7 or caspase-11, but is weakly processed into p18 and p11 subunits by murine caspase-8. No aspartase activity of murine caspase -14 could be generated by bacterial or yeast expression. Transient overexpression of murine caspase-14 in mammalian cells did not elicit cell death and did not interfere with caspase-8-induced apoptosis. In conclusion, caspase-14 is a member of the caspase family but no proteolytic or biological activities have been identified so far. The high constitutive expression levels in embryos and specific expression in adult skin suggest a role in ontogenesis and skin physiology.
\end{abstract}

Keywords: caspase; cloning; apoptosis; ICE; CED-3; cell death

Abbreviations: DD, death domain; d.p.c., days post coitus; EST, expressed sequence tag

\section{Introduction}

Members of the caspase protein family are key mediators in the execution of apoptotic cell death. Specific inhibition of one or more caspases by $\mathrm{CrmA}$, p35 or the peptide derivatives AcYVAD-CHO and Ac-DEVD-CHO revealed that several caspases are involved in apoptosis mediated by stimulated Fas or 55-kDa tumor necrosis factor receptor (Los et al, 1995; Bertin et al, 1997; Nagata, 1997). Both receptors transduce the death signal through a cytoplasmic sequence motif called 'death domain' (DD). After receptor trimerization this domain rapidly associates with a similar DD in the adapter molecules TRADD and/or FADD. The N-terminal end of FADD exhibits significant sequence homology to two similar regions within the prodomain of caspase-8 and caspase-10, referred to as death effector domains, which allow heterodimerization of these caspases with FADD (Boldin et al, 1996; Muzio et al, 1996; Vincenz and Dixit, 1997). In the case of Fas, it has been demonstrated that receptor occupation results in FADDmediated recruitment of caspase-8 into the receptor complex. Receptor-associated caspase-8 is then proteolytically cleaved to generate active caspase-8 (Yang et al, 1998). Once caspase- 8 has become activated, it might initiate a proteolytic caspase activation cascade, since it is able to process, at least in vitro, all known caspases into their active subunits (Srinivasula et al, 1996). Activated caspases are believed to be the executors of cell death by aspartatespecific proteolysis of substrates, which results in the characteristic features of apoptosis, such as DNA degradation, nuclear condensation and membrane blebbing (Villa et al, 1997).

Here we report the identification of a new member of the caspase gene family, viz. caspase-14, which might be involved in ontogenesis and skin physiology.

\section{Results and Discussion}

\section{Identification of murine and human caspase-14}

TBLASTN homology searches in the expressed sequence tag (EST) database, with human caspases as a query, revealed two murine ESTs coding for an unidentified caspase-like protein, viz. the 15.5 days post coitus (d.p.c.), embryonalderived cDNA clone 555962, and the 11-week adult mouse whole skin-derived cDNA clone 607978. Sequencing of these cDNA clones revealed that both encode the same open reading frame and contain an in-frame stop codon $5^{\prime}$ of the start codon (data not shown). This suggests that these cDNAs span the full open reading frame. Considering that the prodomain of caspases is removed from the p20 subunit by cleavage after Asp, murine caspase-14 may contain either no prodomain or a prodomain of $7\left(D_{7}\right)$ or $17\left(D_{17}\right)$ amino acids. However, based on multiple sequence alignment (data not shown) the Tyr residue at position $16\left(\mathrm{Y}_{16}\right)$ is conserved in the large subunit of all known mammalian caspases. If there is a 


\section{A}

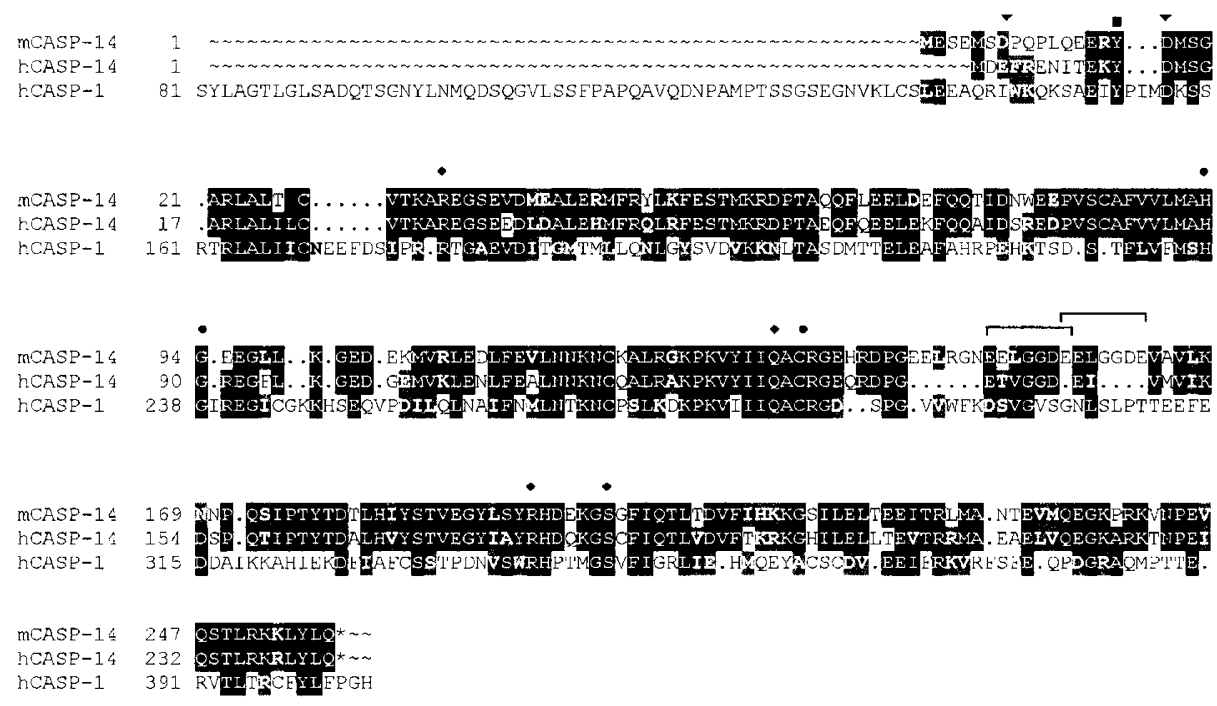

B

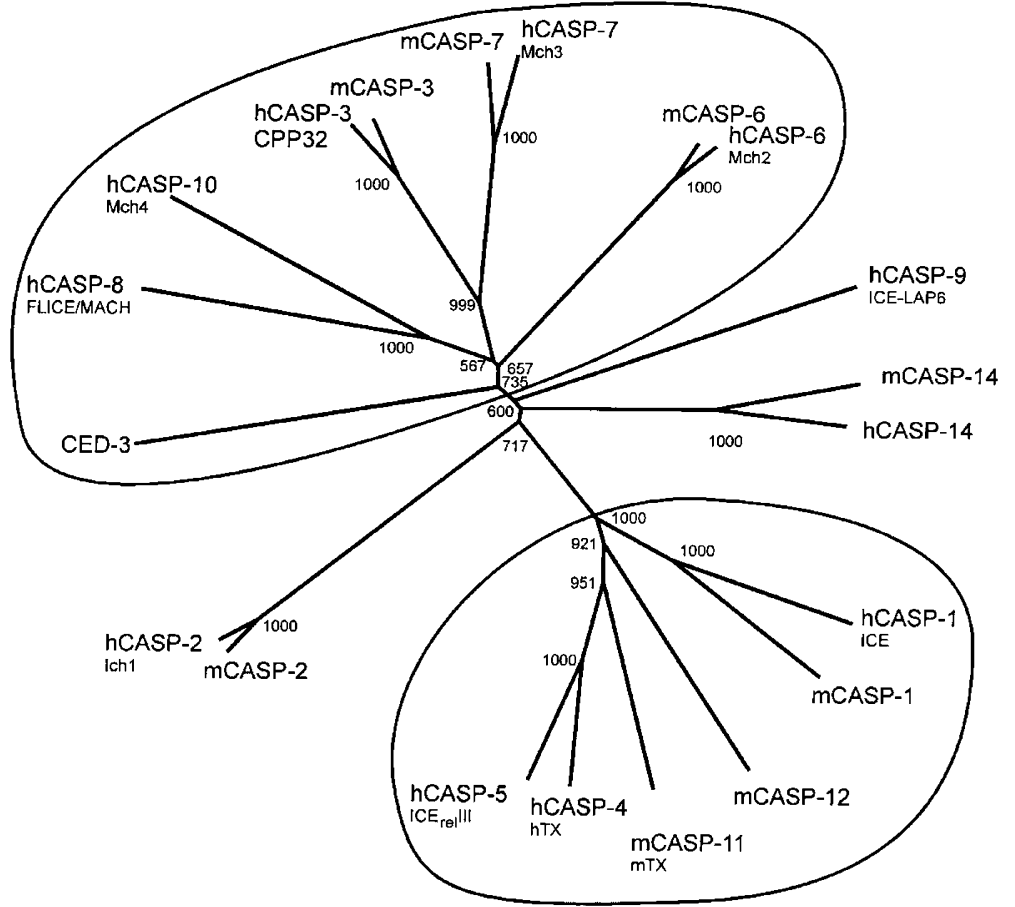

Figure 1 Analysis of caspase-14 sequences and their relationship with the caspase family. (A) Alignment of the amino acid sequence of murine and human caspase-14 with human caspase-1. The murine caspase-14 sequence was obtained by sequencing the EST clones 555962 and 607978 . The sequence of human caspase-14 is predicted from cosmid R31973 (Ac004699) by the GENSCAN computer program. Identical and similar amino acids are boxed in black and gray, respectively. amino acids aligning with the residues in human caspase- 1 which form the binding pocket for P1 Asp., amino acids involved in catalysis. $\mathbf{\square}$, $\mathrm{Y}_{16}$ believed to belong to the p18 subunit. $\boldsymbol{\nabla}, D_{7}$ and $D_{17}$ define a putative prodomain and a less likely alternative, respectively. Brackets indicate both EELGGDE heptapeptides containing the presumed cleavage sites between p18 and p11 subunits of murine caspase-14. In human caspase-14 only one of these potential cleavage sites is conserved. (B) Phylogenetic comparison of human and murine caspases. The amino acid sequences were aligned using the Genetics Computer Group (Madison, WI, USA) PILEUP algorithm. This alignment was inserted into the CLUSTAL W program (Thompson et al, 1994) to generate the dendrogram. Boottrap values are indicated at the fork of each branch. The well-defined caspase- 1 and caspase- 3 subfamily members are encircled. Note that murine caspase11 is presumably the homologue of human caspase-4 (TX) (Van de Craen et al, 1997a) 


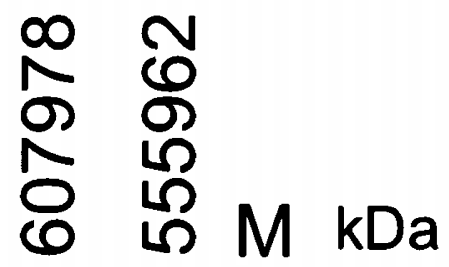

$-46$

$\triangle \mathrm{mCASP}-14$

$-30$

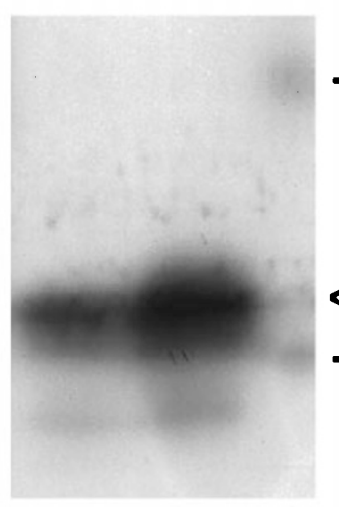

A

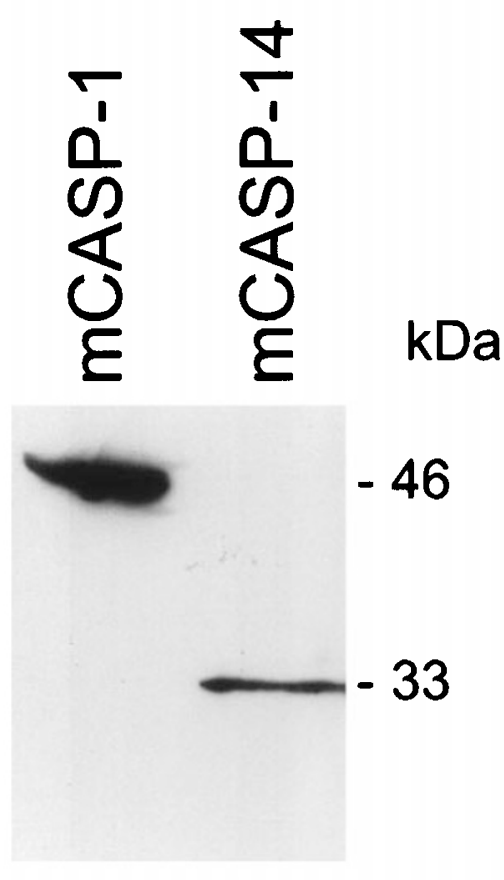

B

Figure 2 Polypeptide length verification of murine caspase-14. (A) In vitro transcription and translation of murine caspase-14 clones in reticulocyte lysate in the presence of ${ }^{35} \mathrm{~S}$-methionine for $90 \mathrm{~min}$ at $30^{\circ} \mathrm{C} .1 \mu \mathrm{l}$ of this mixture was loaded on a $15 \%$ SDS-polyacrylamide gel. $\mathrm{M}, \mathrm{M}_{\mathrm{r}}$ marker. (B) Detection of $\mathrm{E}$ tagged caspase-14 in HEK293T lysates. HEK293T cells were transiently transfected with E-tagged murine caspase-1 or murine caspase-14. The lysates were prepared $30 \mathrm{~h}$ later, fractionated by SDS-PAGE, electroblotted and revealed with an anti-E-tag antibody
A

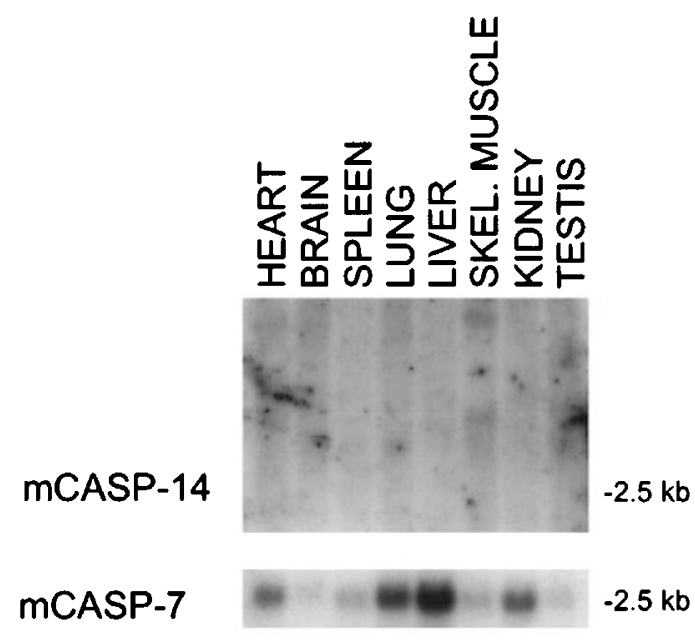

B

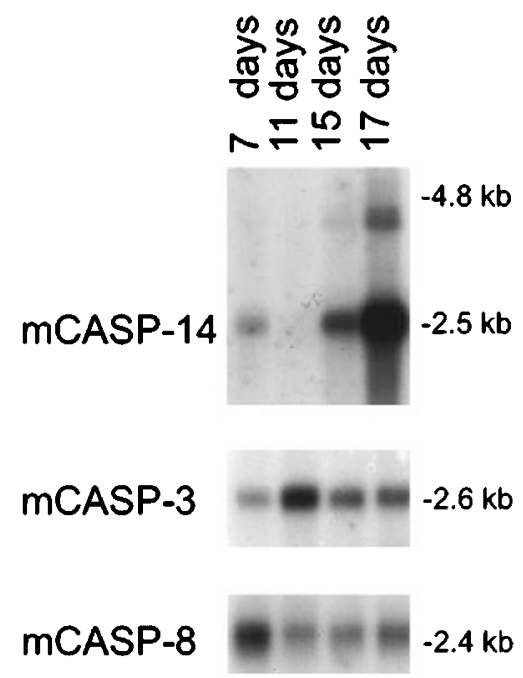

C

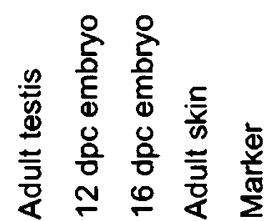

mCASP-14

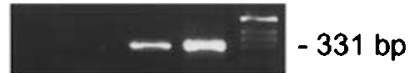

ACTIN

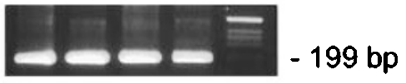

Figure 3 Expression pattern of murine caspase-14. (A) Mouse multiple tissue Northern blot hybridized sequentially with murine caspase-14 or murine caspase-7 cDNA. Each lane contains $2 \mu \mathrm{g}$ poly $(\mathrm{A})^{+}$RNA. (B) Whole mouse embryo Northern blot hybridized with murine caspase-14, -3 or -8 cDNA. Each lane contains $2 \mu \mathrm{g}$ poly $(\mathrm{A})^{+}$RNA of whole embryos in different stages of development, as shown on top. (C) Reverse transcriptase PCR on total RNA derived from murine adult testis, 12 d.p.c. murine embryo, 16 d.p.c. murine embryo and murine adult skin. At the top, amplifications performed with caspase-14 specific primers. At the bottom, amplifications performed with actin specific primers 
prodomain, $D_{7}$ is the most likely candidate. Three Asp residues $\left(D_{142}, D_{156}\right.$ and $\left.D_{162}\right)$ are potential cleavage sites between a p18 and a p11 subunit. His ${ }_{93}$, Gly $_{94}$ and $\mathrm{Cys}_{136}$, homologous to the catalytically important residues of human caspase-1, are conserved (Walker et al, 1994; Wilson et al, 1994) (Figure 1A). The amino acids $\operatorname{Arg}_{33}, \mathrm{Gln}_{134}, \mathrm{Arg}_{294}$ and Ser $_{200}$, constituting the Asp-binding pocket, are the same as in human caspase-1 (Walker et al, 1994; Wilson et al, 1994)

A<smiles>C[As](C)O[W]</smiles>

$\triangleleft(\mathrm{His})_{6} \mathrm{mCASP}-14$ $-30$

$-14.6$

\begin{tabular}{|c|c|c|c|}
\hline (D7) & $\begin{array}{l}\text { D162 } \\
\text { D156 }\end{array}$ & $\mathrm{T}$ & Ex| \\
\hline p18 & 11p p11 & 31.4 & \\
\hline is $\left.s_{6}\right)$ & E163 & & \\
\hline (D7) & $\begin{array}{l}p 11 \\
\end{array}$ & 11.5 & 11 \\
\hline \begin{tabular}{|l|l}
$p 18$ \\
\end{tabular} & & 20 & 24 \\
\hline
\end{tabular}

B

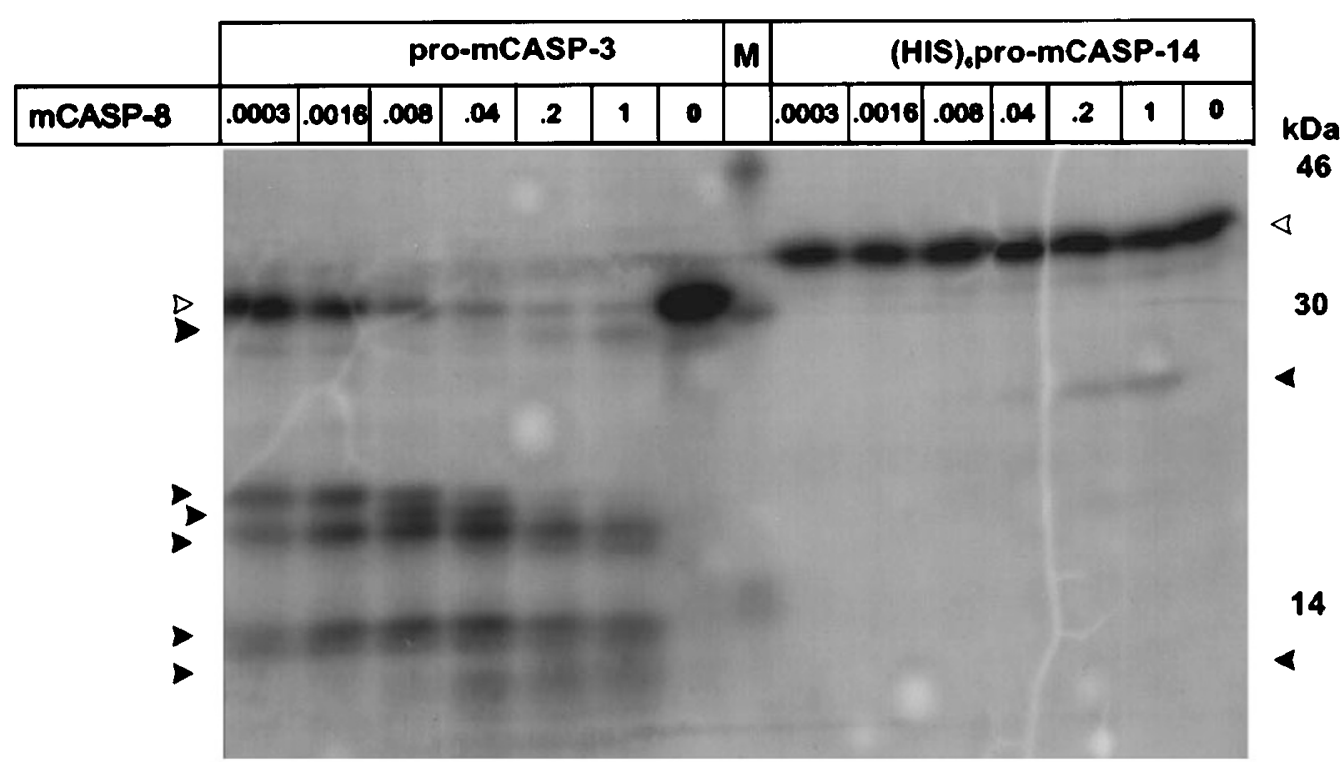

Figure 4 In vitro cleavage of ${ }^{35} \mathrm{~S}$-methionine-labeled $\mathrm{His}_{6}$-tagged murine caspase-14 by murine caspase-8. (A) $5 \mu$ l of in vitro labeled, purified His ${ }_{6}$-tagged murine caspase-14 was incubated with $30 \mu \mathrm{g}$ lysate of Escherichia coli transfected with pLT-mCASP-8 plasmid. Lysate of bacteria transfected with empty pLT10TH served as a control. The resulting cleavage products are shown on the right. $\mathbf{n}$. His $s_{6} \operatorname{tag}$ of $2 \mathrm{kDa} ; \mathrm{T}$, theoretically calculated $M_{\mathrm{r}}$; Exp, experimentally found $M_{\mathrm{r}}$. (B) A $1: 5$ serial dilution was made starting with $30 \mu \mathrm{g}$ lysate of bacteria expressing pLT-mCASP-8 $(=1)$. Each dilution was incubated with $5 \mu$ l of labeled, purified His ${ }_{6}$-tagged murine caspase-14 or $1 \mu \mathrm{l}$ of radiolabeled murine caspase-3. Reaction products were separated on $15 \%$ SDS-PAGE, followed by autoradiography to reveal cleavage fragments. Uncleaved products are indicated by open arrowheads; resulting cleavage fragments are shown by closed arrowheads. $\mathrm{M}, M_{\mathrm{r}}$ marker 
(Figure 1A) and other murine and human caspases (Van de Craen et al, 1997a). These features suggest that caspase-14, like caspase-1, exerts a cysteine protease activity upon activation.

A TBLASTN homology search in the EMBL database, with murine caspase-14 as a query, revealed that human cosmid R31973 (Ac004699) contains a gene with high homology to murine caspase-14. Assembly of the exons of this gene disclosed a putative coding sequence of human caspase-14. The theoretical open reading frame of human caspase-14 shows $72 \%$ identity and $83 \%$ similarity to murine caspase-14 (Figure $1 \mathrm{~A}$ and $\mathrm{B}$ ). Human cosmid R31973 was assigned to chromosome 19p13.1 (Ac004699). Figure 1B shows that murine and human caspase-14 are relatively more related to human caspase- 9 and human/murine caspase-2, and that they are no members of the caspase- 3 or caspase- 1 subfamilies. This suggests that, besides the well-defined caspase-1 and caspase-3 subfamilies, a third group of proteins (human caspase-9, human/murine caspase-2 and human/murine caspase-14) is emerging, with relatively low homology to each other. Therefore, it is doubtful whether they can be considered one subfamily.

In order to verify whether the theoretical length of the murine open reading frame corresponds with the $M_{\mathrm{r}}$ of the protein coded for by the cDNA, both clones 607978 and 555962 were in vitro transcribed and translated in the presence of ${ }^{35} \mathrm{~S}$-methionine. The resulting products were analyzed on 15\% SDS-PAGE. As shown in Figure 2A, both murine caspase-14-encoding clones generated a product of approximately $31 \mathrm{kDa}$ (the theoretical calculated $M_{\mathrm{r}}$ is 29.5), indicating that the presumed start codon is used. In order to confirm that murine caspase-14 is correctly expressed in eukaryotic cells, it was elongated at its $\mathrm{C}$-terminus with an $\mathrm{E}$ tag (pCDNA-mCASP-14E). This expression plasmid was transiently transfected in HEK293T cells and the product length of the heterologously expressed protein was verified by Western blotting with an anti-E-tag antibody. E-tagged murine caspase-1 expression was included as a positive control. As expected, murine caspase-14 and murine caspase-1 were expressed as proteins of $33 \mathrm{kDa}$ and $46 \mathrm{kDa}$, respectively (Figure $2 \mathrm{~B}$ ).

\section{Expression pattern of murine caspase-14}

In two independent experiments a multiple adult tissue Northern blot was hybridized with the 1719 bp EcoR fragment of clone 607978. No hybridization was observed with this caspase-14 probe (Figure $3 \mathrm{~A}$ ). The quality of the blot was checked using a murine caspase-7 cDNA probe. A murine caspase-7 transcript of $2.5 \mathrm{~kb}$ was clearly detectable (Figure 3A) and the expression pattern was similar to that found previously (Van de Craen et al, 1997a). It can be concluded that murine caspase-14 transcripts, if any, were below the detection limit in these adult tissues examined. Since clone 555962 was isolated from a mouse embryo cDNA library, we examined whether murine caspase-14 mRNA was expressed in mouse embryos. A Northern blot was hybridized containing poly $(A)^{+}$RNA from 7, 11, 15 and 17 days old, whole embryos (Figure $3 \mathrm{~B}$ ). The murine caspase-14 probe clearly recognized a transcript of $2.5 \mathrm{~kb}$. The murine caspase14 mRNA expression increased during maturation of the embryo (Figure 3B). Remarkably, the 11-day old embryo did not show expression of caspase-14 mRNA. Hybridization of the same blot with a caspase- 3 or a caspase- 8 probe suggests that all lanes contain equal amounts of poly $(A)^{+}$ RNA (Figure 3B). Because the caspase-14 transcript size, which includes a large poly $(A)^{+}$tail, was consistent with the length of both cDNA clones (2203 and $2213 \mathrm{bp}$ ), the latter presumably contained an insert corresponding to the major caspase-14 mRNA species. A weaker band was detected at $4.5 \mathrm{~kb}$ and possibly represents incompletely or alternatively processed mRNA. Since clone 607978 was isolated from a mouse skin-derived cDNA library, the expression of caspase14 transcripts was examined in adult mouse skin by reverse transcriptase PCR (RT - PCR). Figure $3 \mathrm{C}$ demonstrates that a caspase-14 cDNA fragment could be amplified from RNA prepared from adult mouse skin (lane 4), while RT-PCR performed on RNA from adult mouse testis did not result in amplification of the caspase-14 cDNA fragment (lane 1). To confirm the apparent absence of caspase-14 transcripts around day 11 of development, additional amplifications were performed on RNA isolated from 12 and 16 d.p.c. mouse embryos. Indeed, caspase-14 could be amplified from RNA derived from 16 d.p.c. embryos but not from RNA derived from 12 d.p.c. embryos (Figure 3, lanes 2 and 3). Amplification of an actin cDNA fragment was identical for all RNA preparations (Figure $3 C$ ). In summary, the expression of caspase-14 transcripts increases during maturation of a mouse embryo but drops temporarily to an undetectable level around 11-12 d.p.c. Additionally, the expression pattern in adult tissues is very restricted since only skin expressed caspase-14 transcripts among the adult tissues examined.

\section{Murine caspase-14 is a weak substrate of caspase-8, but is no substrate of caspase-1, caspase-2, caspase-3, caspase-6, caspase-7, caspase-11 or granzyme B}

Members of the caspase family are activated by cleavage at Asp residues to generate p20 and p10 subunits which constitute the active tetrameric enzyme $\left(\mathrm{p} 20_{2} / \mathrm{p} 10_{2}\right)$ (Walker et al, 1994; Wilson et al, 1994; Nicholson et al, 1995). Since proforms of caspases are often substrates for active $\mathrm{p2O}_{2} /$ $\mathrm{p} 10_{2}$ caspases (Srinivasula et al, 1996) or granzyme B (Darmon et al, 1995; Van de Craen et al, 1997b), we examined whether caspase-14 was proteolytically cleaved by these proteases. Therefore, ${ }^{35} \mathrm{~S}$-methionine-labeled caspase-14 was incubated with purified murine caspase- $x$ $(x=1,2,3,6,7$ or 11$)$ or purified murine granzyme $B$. However, none of these active enzyme preparations was able to cleave caspase-14 (results not shown).

Because purified, enzymatically active murine caspase-8 was not available, a lysate of Escherichia coli-expressing pLT- $\Delta$ promCASP-8 was used to examine whether murine caspase-8 exhibited proteolytic activity on in vitro radiolabeled and partially purified $\mathrm{His}_{6}$-tagged murine caspase14. The $\mathrm{His}_{6}$-tagged murine caspase-14 migrated about $4 \mathrm{kDa}$ slower on SDS-PAGE as compared to non-tagged caspase-14 (the epitope tag adds $2 \mathrm{kDa}$, resulting in a 
theoretical $31.4 \mathrm{kDa}$ ). Figure $4 \mathrm{~A}$ shows that murine caspase- 8 cleaved this $35.5 \mathrm{kDa}$ murine caspase-14 weakly into $24 \mathrm{kDa}$ (p18) and $11 \mathrm{kDa}$ (p11) fragments. These products suggest cleavage at $D_{156}$ and/or $D_{162}$. Since these Asp residues are both embedded in an identical, repeated EELGGDE heptapeptide (Figure 1A), it is quite possible that cleavage occurs at both sites. In human caspase-14 only one of these sites is conserved (Figure 1A). It should be noted that the slower migration of the full-length $\mathrm{His}_{6}$-tagged murine caspase-14 was largely due to the charged $\mathrm{His}_{6}$ tag, since the p18 fragment also runs approximately $4 \mathrm{kDa}$ slower than theoretically expected (Figure 4A).

To investigate the efficiency of caspase-8-mediated cleavage of caspase-14, a 1/5 serial dilution of lysate from murine caspase-8-expressing bacteria was incubated with radiolabeled $\mathrm{His}_{6}$-tagged murine caspase-14 or radiolabeled murine caspase- 3 for $1.5 \mathrm{~h}$ at $37^{\circ} \mathrm{C}$. The resulting products were analyzed by SDS-PAGE and autoradiography. Figure $4 \mathrm{~B}$ clearly demonstrates that proteolytic activity of caspase-8 was at least 1000 times more efficient on caspase-3 than on caspase-14. Consequently, it is quite possible that other proteases may process murine caspase-14 more efficiently than caspase-8 or that caspase-14 activation involves unknown mechanisms.

\section{Bacterial expression of murine caspase-14}

Bacterial expression of caspases lacking their prodomain (p30) usually results in the generation of p20 and p10 domains in the soluble fraction of the bacterial extract (Molineaux et al, 1993; Kamens et al, 1995). Three different pLT10TH constructs were made to generate active murine caspase-14 enzyme. One plasmid contained the full-length murine caspase-14 coding sequence (pLT-mCASP-14). Furthermore, two constructs were generated in which a putative prodomain was removed (pLT- $\Delta_{1-7} \mathrm{mCASP}-14$ and pLT- $\left.\Delta_{1-17} \mathrm{CASP}-14\right)$. To facilitate the purification of the proteins, an $\mathrm{N}$-terminal polyhistidine sequence ( $\mathrm{His}_{6}$ tag) was fused to the murine caspase-14 sequences. The same conditions for bacterial expression were used as for the generation of other prodomain-deleted murine caspases. These conditions generated active caspase-1, caspase-2, caspase-3, caspase-6, caspase-7, caspase-8 and caspase11 (Van de Craen et al, in preparation). All caspase-14 constructs were well expressed, but the product was predominantly present in insoluble inclusion bodies. No p18 or p11 subunits were detectable after bacterial expression; neither in the soluble nor in the insoluble fractions was aspartase activity detected using Ac-DEVDAMC $(100 \mu \mathrm{M})$, Ac-YVAD-AMC $(100 \mu \mathrm{M})$ or z-VAD-AFC $(100 \mu \mathrm{M})$ fluorogenic peptide caspase substrates. This was also the case after $30 \mathrm{~min}$ preincubation of the soluble fraction at $37^{\circ} \mathrm{C}$, which might have allowed an autocatalytic generation of caspase activity (Ramage et al, 1995). Also denaturation and refolding of the insoluble fractions of murine caspase-14 or separate expression of the subunits did not generate activity on the fluorogenic tetrapeptide substrates (data not shown).

\section{Murine caspase-14 does not autoprocess in yeast}

We have previously successfully used a yeast expression system to demonstrate autoprocessing of murine caspase-1 (Van Criekinge et al, 1996). Since bacterial expression of murine caspase-14 suggests the inability of this caspase to autoprocess, the autocleavage activity of murine caspase-14 was tested in a yeast expression system. pAS2 plasmids encoding the Gal4 DNA-binding domain (Gal4DB) fused to murine caspase-1, murine caspase-1(C285S), murine caspase-14 or murine caspase-14(C136S) were transformed in Saccharomyces cerevisiae strain YRG-2. Single yeast colonies were used to prepare lysates for Western blot analysis with antibodies against Gal4DB. However, also in yeast no autoprocessing activity could be detected for wildtype murine caspase-14 (Figure 5). Transformations with caspase-1 or the inactive Cys mutant CASP-1(C285S) fused to Gal4DB were used as controls and confirmed that the $p 10$ subunit of caspase-1 was cleaved off without involvement of endogenous yeast proteases (Figure 5). These results indicate that murine caspase-14, in contrast to murine caspase-1, is not able to autoprocess in $S$. cerevisiae, which might contain eukaryotic helping factors. However, it should be kept in mind that caspase-14 contains no large prodomain that can be involved in oligomerization-induced autoactivation, as has been demonstrated for caspase-1 (Van Criekinge et al, 1996) and caspase-8 (Yang et al, 1998).

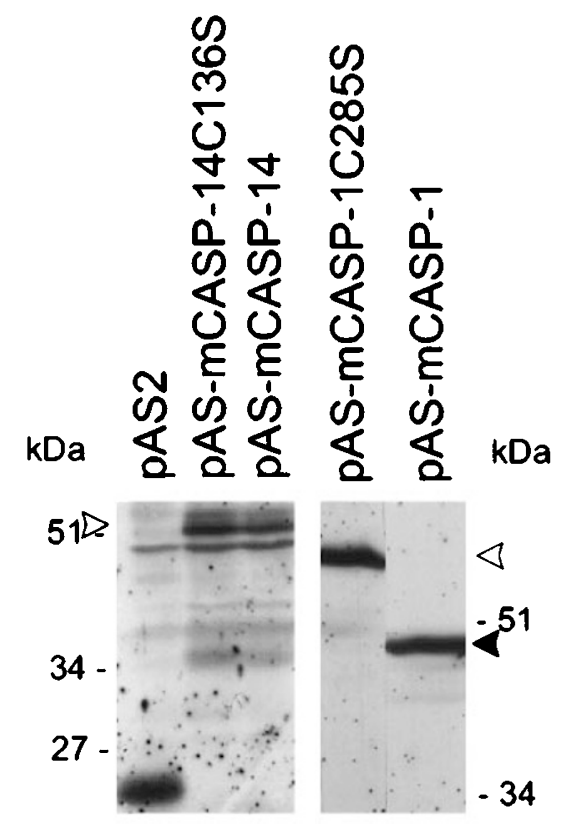

Figure 5 Murine caspase-14 does not autoprocess in S. cerevisiae. Lysates of $S$. cerevisae strain YRG-2, transformed with pAS2-encoding Gal4DB fusions with full-length murine caspase-1, murine caspase-1(C285S), murine caspase-14 or murine caspase-14(C136S), were subjected to SDS-PAGE, electroblotted and revealed with anti-Gal4DB antibody. In the lane at the right hand side, the cleavage product of the Gal4DB fusion with murine caspase- 1 is derived from proteolysis at the C-terminal p10 domain of murine caspase-1 
Transiently transfected murine caspase-14 exhibits no evident apoptotic-related activity in mammalian cells

Caspase-14 was examined for its capacity to induce cell death by transient overexpression in HeLaH21 cells. These cells were transfected with three different vectors expressing caspase-14, viz. pCDNA-mCASP-14E (Etagged caspase-14), pCAG-mCASP-14 or pCAG-mCASP14C136S. The extent of cell death in the transfected population was measured by the decrease in number of adherent $\beta$ Gal-expressing cells as revealed using an XGal substrate. A pCDNAl-derived expression vector coding for FADD was used as a positive control. Figure $6 \mathrm{~A}$ shows that transient overexpression of the FADD-containing plasmid caused a profound reduction in number of $\beta$ Gal-expressing HeLaH21 cells as compared to the negative control (pCDNAI). Transient overexpression of the different murine caspase-14-encoding constructs did not result in a clear reduction in number of adherent blue cells (Figure 6A), suggesting that murine caspase-14 itself is not capable to induce apoptosis. Similar results were obtained in HEK293T cells.

A

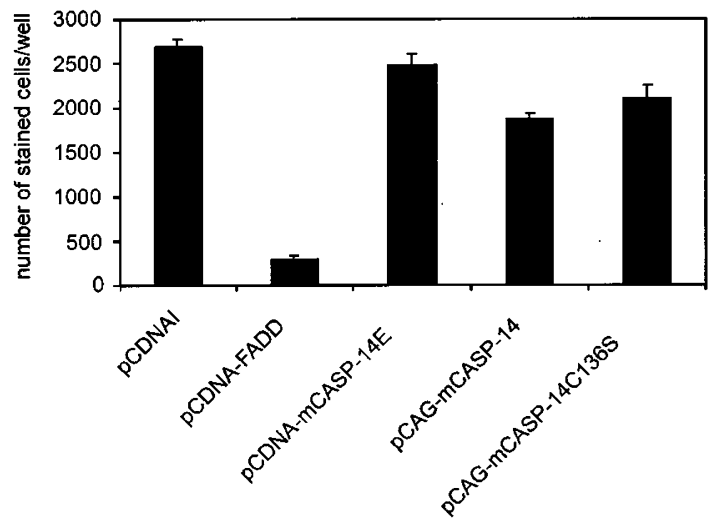

B

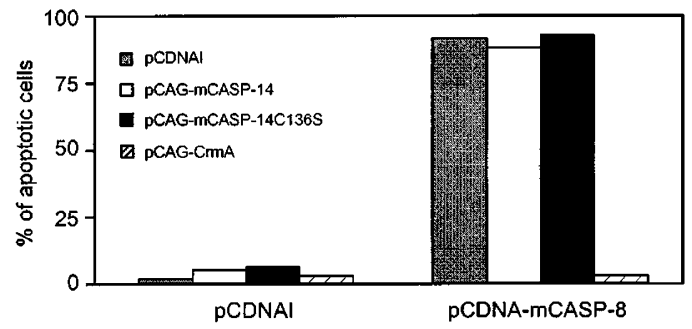

Figure 6 Murine caspase-14 has no clear function in apoptosis. (A) HeLaH21 cells were cotransfected with pUT651, pCDNA-FADD, pCDNAmCASP-14E, pCAG-mCASP-14, pCAG-mCASP-14C136S or empty pCDNAI. After $24 \mathrm{~h}$, cells were stained with $X G$ al and counted. No clear apoptotic morphology was observed after transfection with a caspase-14-expressing construct. (B) HEK293T cells were transfected with $200 \mathrm{ng}$ pUT651 (for visualization of transfected cells), $50 \mathrm{ng}$ pCDNA-mCASP-8 (for induction of cell death) and $600 \mathrm{ng}$ pCAG-mCASP-14, pCAG-mCASP-14C136S, pCAGCrmA or empty pCDNAI. Controls included experiments with $50 \mathrm{ng}$ pCDNAI instead of pCDNA-mCASP-8. After $24 \mathrm{~h}$, cells were stained with XGal. Since HEK293T cells were very efficiently transfected, the percentage of blue apoptotic cells was determined
With FLIP (Irmler et al, 1997) as an example, it is conceivable that also caspase-14 exerts apoptosis-inhibiting features. Another link to a potential caspase-14interfering activity is the low protease activity of caspase8 on caspase- 14 as revealed by in vitro cleavage assays. Since Western blot analysis revealed that murine caspase14 is well expressed in HEK293T cells, and since murine caspase-8 induced clear apoptosis in this cell line (Van de Craen et al, unpublished observations), the potential effect of murine caspase-14 on murine caspase-8-induced apoptosis was examined in HEK293T cells by transient cotransfection experiments. Cotransfection of caspase-8 with CrmA was applied as a positive control for inhibition. Figure 6B demonstrates that caspase-8-induced cell death was not influenced by cotransfection with caspase-14 or caspase-14(C136S), while CrmA inhibited cell death significantly. Also dilution series of a murine caspase-8encoding plasmid, cotransfected with caspase-14expressing constructs, did not reveal any effect of caspase-14 (data not shown). These data indicate that caspase-14 does not interfere negatively with apoptosis, at least when cell death is induced by caspase-8.

In conclusion, we have identified a new caspase homologue, caspase-14. Although the amino acid sequence would classify murine caspase-14 as a bona fide cysteine aspartase, no aspartase activity could be revealed. Further experiments, such as generation of caspase-14-deficient mice and in situ hybridizations, will be needed to assign proper biological functions to this new caspase homologue. Nevertheless, the expression of caspase-14 mRNA in whole embryos and in adult skin suggests a role in ontogenesis and skin physiology.

\section{Materials and Methods}

\section{Cloning of murine caspase-14}

In order to identify new murine caspases, TBLASTN searches for homology with known human caspases were performed on an EST database. Two new murine cDNA clones with significant caspase homology were identified, viz. clones 555962 and 607978. The physical cDNA clones were obtained from the I.M.A.G.E. consortium (Washington University School of Medicine, St. Louis, MO, USA; Lennon et al, 1996). Sequencing of the entire inserts on an ABI373A sequencer (Applied Biosystems, Foster City, CA, USA) revealed that clones 555962 and 607978 encoded the same full open reading frame, called caspase- 14 .

\section{Identification of human caspase-14}

A TBLASTN search in the EMBL database, with murine caspase-14 as a query, revealed that cosmid R31973 (Ac004699) contains the human caspase-14 gene. The GENSCAN computer program was applied to deduce the coding sequences. GENSCAN predicts that the human caspase-14-coding sequence is $729 \mathrm{bp}$ long and encodes a protein of 242 amino acids. The theoretical $M_{r}$ is 27.7 .

\section{Plasmid constructions}

Clone 555962 was used as a template to amplify caspase-14 with the PCR primers GCGAAGCTTCCACCATGGAGTCAGAGATGAGT- 
GATCCT and GGGAGAAGCGGCCGCTTGCAAATAGAGCTTCTTCC. The Hindlli/Notl-digested amplicon was provided with a C-terminal Etag in a modified pCDNAI vector (Invitrogen, San Diego, CA, USA) to generate pCDNA-mCASP-14E. To construct pCAG-mCASP-14, murine caspase-14 was amplified with the primers GCGGATATCCACCATGGAGTCAGAGATGAGTGATCCT and GCGGATATCTTATTGCAAATAGAGCTTCTTCC. Then the EcoRV-digested amplicon was ligated in a Ball-opened pCAGGS expression vector (Niwa et al, 1991). The cowpox CrmA gene (a generous gift from Dr. D. Pickup, Durham, NC, USA) was EcoRI-cloned in PCAGGS, resulting in pCAG-CrmA. The bacterial expression vectors for caspase-14 were constructed by $P C R$ with GCGGATATCCATGGAGTCAGAGATGAGTGATCCT (F1), GCGGATATCACCTCAGCCATTGCAGGAGGAAAGA (F2), GCGGATATCCATGTCAGGTGCCCGCCTGGCCCTGACG (F3) and GCGGAATTCGATATCTTATTGCAAATAGAGCTTCTTCC (R). Primers F1 and $R$ amplified full-length caspase-14, primers $F 2$ and $R$ created the putative prodomain-deleted $\Delta_{1-7} \mathrm{mCASP}-14$, and primers $\mathrm{F} 3$ and $\mathrm{R}$ amplified $\Delta_{1-17} \mathrm{mCASP}-14$. The products were EcoRV-cloned into a pLT10TH vector (Mertens et al, 1995), resulting in pLT-mCASP-14, pLT- $\Delta_{1-7}$ mCASP-14 and pLT- $\Delta_{1-17}$ mCASP-14, respectively. The caspase-14 inserts were $\mathrm{N}$-terminally fused to a $\mathrm{His}_{6}$ tag to facilitate purification. The same cloning strategy with the primers GCGGATATCCAGTGAGTCACGGACTTCAGACAAAG and GCGGATATCGAATTCTCATTAGGGAGGGAAGAAGAGCTTC (and EST clone 533745 as template DNA) was applied to generate the bacterial expression vector for prodomain-deleted murine caspase-8, viz. pLT- $\Delta$ promCASP-8.

Yeast murine caspase-14 expression plasmids were also constructed by PCR. The primers used were: AAAGAGATCGAATTCGCCATGGAGTCAGAGATGAGTGATCCTC (YF), ATAGATCTCTGCAGGTCGACGTTATTGCAAATAGAGCTTCTTCCGGAG (YR), CATCCAGGCTAGTAGAGGAGAGCACAGAG (YCSF) and CTCTGTGCTCTCCTCTACTAGCCTGGATG (YCSR). Primers YF and YR were used to generate wild-type caspase-14. The four primers were applied to mutate the active Cys residue to Ser (C136S). All amplicons were $\mathrm{Ncol} / \mathrm{Sall}$-inserted in pAS2, to result in pASmCASP14 and pASmCASP-14C136S. The Gal4DBp45ICE-C285S plasmid (Van Criekinge et al, 1996) was renamed pASmCASP-1C285S.

\section{Northern blot analysis}

A multiple tissue Northern blot of eight different mouse adult tissues and a Northern blot containing poly $(\mathrm{A})^{+} \mathrm{RNA}$ from four mouse embryos (7, 11, 15 and 17 days old) were purchased from Clontech Laboratories (Palo Alto, CA, USA). Sequential hybridization and stripping were performed according to the manufacturer's instructions.

\section{Reverse transcriptase PCR}

Reverse transcriptase reactions on total RNA isolated from murine adult skin, murine adult testis, 12 d.p.c. murine embryos and 16 d.p.c. murine embryos were performed by the superscript preamplification system for first-strand cDNA synthesis (Gibco BRL, Rockville, MD, USA). The primers GCGGATATCTGAGGTTGCTGTGCTCAAGAACAACC and GCGGAATTCGATATCTTATTGCAAATAGAGCTTCTTCC were used to amplify a caspase-14 cDNA fragment of $331 \mathrm{bp}$, while the primers GCTCACCATGGATGATGATATCGCC and GGATGCCTCTCTTGCTCTGGGCCTC were applied for amplification of an actin cDNA fragment of $199 \mathrm{bp}$.

\section{Cell lines and transient transfections}

HeLaH21 is a human cervix carcinoma derived from HeLa cells. HEK293T is a human embryonal kidney carcinoma stably transfected with the SV40 T-antigen, having a transfection efficiency of $>40 \%$ (a generous gift of Dr. M. Hall, University of Birmingham, UK; DuBridge et al, 1987). In order to assess the size of epitope-tagged murine caspase-14, $5 \times 10^{5}$ HEK293T cells/6-well were transiently transfected using the calcium phosphate precipitation method (O'Mahoney and Adams, 1994). A total amount of $1 \mu \mathrm{g}$ plasmid DNA was used. Cell lysates were prepared with lysis buffer containing $50 \mathrm{mM}$ Tris $\mathrm{pH}$ 8.0, $300 \mathrm{mM} \mathrm{NaCl}, 5 \mathrm{mM}$ EDTA, $15 \mathrm{mM} \mathrm{MgCl}$, 1\% NP-40, 1 mM PMSF, $50 \mu \mathrm{M}$ leupeptin and $20 \mu \mathrm{g} / \mathrm{ml}$ aprotinin. $90 \mu \mathrm{g}$ of total protein was used for Western analysis with anti-E-tag antibody (Pharmacia Biotech, Uppsala, Sweden).

In order to evaluate the apoptotic activity of caspases, $5 \times 10^{5}$ HeLaH21 cells/6-well were transiently transfected with DOPE transfection reagent according to the manufacturer's instructions (Eurogentec, Seraing, Belgium). A total of $2 \mu \mathrm{g}$ DNA, 50\% pUT651 (Cayla, Toulouse, France) and 50\% vector of interest [pCAG-mCASP14, pCAG-mCASP-14C136S, pCDNA-mCASP-14E, pCDNA-FADD or empty pCDNAI vector] was used. After $24 \mathrm{~h}$, the cells were tested for cell death by a decrease in number of positive cells using XGal substrate. This $\beta$ Gal assay was performed as described previously (Van de Craen et al, 1997a).

In order to assay the effect of murine caspase-14 on murine caspase-8-induced apoptosis, $5 \times 10^{5}$ HEK293T cells/6-well were transiently transfected using the calcium phosphate precipitation method (O'Mahoney and Adams, 1994). Three plasmids were transfected simultaneously: $200 \mathrm{ng}$ pUT651, $50 \mathrm{ng}$ pCDNA-mCASP8 (which contains full-length murine caspase-8; Van de Craen et al, unpublished observations) and $600 \mathrm{ng}$ pCAG-mCASP-14, pCAGmCASP-14C136S, pCAG-CrmA, or empty pCDNAI. Controls included experiments with empty pCDNAI instead of pCDNA-mCASP-8.

\section{In vitro cleavage assays}

${ }^{35}$ S-methionine-labeled substrates were generated by in vitro transcription and translation as previously described (Van de Craen et al, 1997a). A lysate of bacteria expressing murine caspase-8 was used as a source for this enzyme. $30 \mu \mathrm{g}$ total lysate was incubated with $5 \mu \mathrm{l}$ purified, labeled $\mathrm{His}_{6}$-tagged murine caspase-14 in $20 \mu \mathrm{l}$ caspase buffer for $90 \mathrm{~min}$ at $37^{\circ} \mathrm{C}$. Radiolabeled $\mathrm{His}_{6}$-tagged murine caspase-14 was obtained by in vitro transcription/translation of the pLT-mCASP-14 plasmid and purified using a $\mathrm{Co}^{+2}$ resin (Talon; Promega Biotec, Madison, WI, USA). The substrate specificity of murine caspase- 8 proteolytic activity was determined by making a $1 / 5$ serial dilution of total lysate of murine caspase-8-expressing bacteria starting with $30 \mu \mathrm{g}$ protein. These dilutions were incubated with $5 \mu \mathrm{l}$ purified, radiolabeled, His ${ }_{6}$-tagged caspase-14 or $1 \mu \mathrm{l}$ radiolabeled caspase-3 in a total volume of $20 \mu \mathrm{l}$ for $90 \mathrm{~min}$ at $37^{\circ} \mathrm{C}$.

\section{Bacterial expression}

pLT10-mCASP-14, pLT- $\Delta_{1-7}$ CASP-14 and pLT- $\Delta_{1-17}$ CASP-14 were transformed in MC1061 bacteria containing a pICA2 plasmid (Mertens et al, in preparation). A $50 \mathrm{ml}$ Luria broth culture of $A_{600}=0.5$ was induced overnight with $1 \mathrm{mM} \mathrm{IPTG}$ at $20^{\circ} \mathrm{C}$. The cells were harvested by centrifugation. MC1061 cell pellets were suspended in $1 \mathrm{ml}$ bacterial lysis buffer $(20 \mathrm{mM}$ Tris- $\mathrm{HCl} \mathrm{pH} \mathrm{7.5,10 \%} \mathrm{glycerol,} 1 \mathrm{mM}$ oxidized glutathione, $1 \mathrm{mM}$ PMSF, $50 \mu \mathrm{M}$ leupeptin and $20 \mu \mathrm{g} / \mathrm{ml}$ aprotinin) and lysed by sonication. The soluble and insoluble fractions were separated by centrifugation for $20 \mathrm{~min}$ at $16000 \times \mathrm{g}$. The expressed proteins were analyzed by $12.5 \%$ SDS-PAGE and Coomassie blue staining. The same procedure was followed for pLT- $\Delta$ promCASP-8. 


\section{Autocleavage in yeast}

These experiments were performed as previously described (Van Criekinge et al, 1996), except that the YRG-2 yeast strain (Stratagene Cloning Systems, La Jolla, CA, USA) was used instead of HF7c.

\section{Acknowledgements}

G.V.L. is a Fellow and S.P. a Postdoctoral Researcher with the Vlaams Instituut voor de Bevordering van het Wetenschappelijk-technologisch Onderzoek in de Industrie, respectively. The authors thank Dr. J. Tschopp and Dr. M. Irmler for providing granzyme B and Dr. D. Huylebroeck for fruitful discussions; A. Meeus and M. Vandenhoute are acknowledged for technical assistance. Research was supported by the Interuniversitaire Attractiepolen, a grant No. 9005097N of the Fonds voor Wetenschappelijk Onderzoek-Vlaanderen, and an EC Biomed Program grant No. BMH4CT96-0300.

\section{References}

Bertin J, Armstrong RC, Ottilie S, Martin DA, Wang Y, Banks S, Wang G-H, Senkevich TG, Alnemri ES, Moss B, Lenardo MJ, Tomaselli KJ and Cohen JI (1997) Death effector domain-containing herpesvirus and poxvirus proteins inhibit both Fasand TNFR1-induced apoptosis. Proc. Natl. Acad. Sci. USA 94: 1172-1176

Boldin MP, Goncharov TM, Goltsev YV and Wallach D (1996) Involvement of MACH, a novel MORT1/FADD-interacting protease, in Fas/APO-1- and TNF receptorinduced cell death. Cell 85: 803-815

Darmon AJ, Nicholson DW and Bleackley RC (1995) Activation of the apoptotic protease CPP32 by cytotoxic T-cell-derived granzyme B. Nature 377: 446-448

DuBridge RB, Tang P, Hsia HC, Leong P-M, Miller JH and Calos MP (1987) Analysis of mutation in human cells by using an Epstein-Barr virus shuttle system. Mol. Cell. Biol. 7: 379-387

Irmler M, Thome M, Hahne M, Schneider P, Hofmann K, Steiner V, Bodmer J-L, Schröter M, Burns K, Mattmann C, Rimoldi D, French LE and Tschopp J (1997) Inhibition of death receptor signals by cellular FLIP. Nature 388: 190-195

Kamens J, Paskind M, Hugunin M, Talanian RV, Allen H, Banach D, Bump N, Hackett M, Johnston CG, Li P, Mankovich JA, Terranova M and Ghayur T (1995) Identification and characterization of $\mathrm{ICH}-2$, a novel member of the interleukin$1 \beta$-converting enzyme family of cysteine proteases. J. Biol. Chem. 270: 1525015256

Lennon G, Auffray C, Polymeropoulos M and Soares MB (1996) The I.M.A.G.E. Consortium: An integrated molecular analysis of genomes and their expression. Genomics 33: 151-152

Los M, Van de Craen M, Penning LC, Schenk H, Westendorp M, Baeuerle PA, Dröge W, Krammer PH, Fiers W and Schulze-Osthoff K (1995) Requirement of an ICE/ CED-3 protease for Fas/APO-1-mediated apoptosis. Nature 375: 81-83

Mertens N, Remaut E and Fiers W (1995) Versatile, multi-featured plasmids for highlevel expression of heterologous genes in Escherichia coli: Overproduction of human and murine cytokines. Gene 164: 9-15

Molineaux SM, Casano FJ, Rolando AM, Peterson EP, Limjuco G, Chin J, Griffin PR, Calaycay JR, Ding GJ-F, Yamin T-T, Palyha OC, Luell S, Fletcher D, Miller DK Howard AD, Thornberry NA and Kostura MJ (1993) Interleukin $1 \beta$ (IL-1 $\beta$ ) processing in murine macrophages requires a structurally conserved homologue of human IL-1 $\beta$ converting enzyme. Proc. Natl. Acad. Sci. USA 90: 1809-1813
Muzio M, Chinnaiyan AM, Kischkel FC, O'Rourke K, Shevchenko A, Ni J, Scaffidi C, Bretz JD, Zhang M, Gentz R, Mann M, Krammer PH, Peter ME and Dixit VM (1996) FLICE, a novel FADD homologous ICE/CED-3-like protease, is recruited to the CD95 (Fas/Apo-1) death-inducing signaling complex. Cell 85: 817-827

Nagata S (1997) Apoptosis by death factor. Cell 88: 355-365

Nicholson DW, Ali A, Thornberry NA, Vaillancourt JP, Ding CK, Gallant M, Gareau Y, Griffin PR, Labelle M, Lazebnik YA, Munday NA, Raju SM, Smulson ME, Yamin TT, Yu VL and Miller DK (1995) Identification and inhibition of the ICE/CED-3 protease necessary for mammalian apoptosis. Nature 376: $37-43$

Niwa H, Yamamura K and Miyazaki J (1991) Efficient selection for high-expression transfectants with a novel eukaryotic vector. Gene 108: 193-200

O'Mahoney JV and Adams TE (1994) Optimization of experimental variables influencing reporter gene expression in hepatoma cells following calcium phosphate transfection. DNA Cell Biol. 13: 1227-1232

Ramage P, ChenevalD, Chvei M, Graff P, Hemmig R, Heng R, KocherHP, Mackenzie A, Memmert K, Revesz L and Wishart W (1995) Expression, refolding, and autocatalytic proteolytic processing of the interleukin- $1 \beta$-converting enzyme precursor. J. Biol. Chem. 270: 9378-9383

Srinivasula SM, Ahmad M, Fernandes-Alnemri T, Litwack G and Alnemri ES (1996) Molecular ordering of the Fas-apoptotic pathway: The Fas/APO-1 protease Mch5 is a CrmA-inhibitable protease that activates multiple Ced-3/ICE-like cysteine proteases. Proc. Natl. Acad. Sci. USA 93: 14486-14491

Thompson JD, Higgins DG and Gibson TJ (1994) CLUSTAL W: Improving the sensitivity of progressive multiple sequence alignment through sequence weighting, position-specific gap penalties and weight matrix choice. Nucleic Acids Res. 22: 4673-4680

Van Criekinge W, Beyaert R, Van de Craen M, Vandenabeele P, Schotte P, De Valck D and Fiers W (1996) Functional characterization of the prodomain of interleukin$1 \beta$-converting enzyme. J. Biol. Chem. 271: 27245-27248

Van de Craen M, Vandenabeele P, Declercq W, Van den brande I, Van Loo G, Molemans F, Schotte P, Van Criekinge W, Beyaert R and Fiers W (1997a) Characterization of seven murine caspase family members. FEBS Lett. 403: 61-69

Van de Craen M, Van den brande I, Declercq W, Irmler M, BeyaertR, Tschopp J, Fiers $W$ and Vandenabeele $P$ (1997b) Cleavage of caspase family members by granzyme B: A comparative study in vitro. Eur. J. Immunol. 27: 1296-1299

Villa P, Kaufmann SH and Earnshaw WC (1997) Caspases and caspase inhibitors. Trends Biochem. Sci. 22: 388-393

Vincenz C and Dixit VM (1997) Fas-associated death domain protein interleukin-1 $\beta$ converting enzyme 2 (FLICE2), an ICE/Ced-3 homologue, is proximally involved in CD95- and p55-mediated death signaling. J. Biol. Chem. 272: 6578-6583

Walker NPC, Talanian RV, Brady KD, Dang LC, Bump NJ, Ferenz CR, Franklin S, Ghayur T, Hackett MC, Hammill LD, Herzog L, Hugunin M, Houy W, Mankovich JA, McGuiness L, Orlewicz E, Paskind M, Pratt CA, Reis P, Summani A, Terranova M, Welch JP, Xiong L, Möller A, Tracey DE, Kamen R and Wong WW (1994) Crystal structure of the cysteine protease interleukin-1 $\beta$-converting enzyme: A (p20/p10) 2 homodimer. Cell 78: 343-352

Wilson KP, Black JA, Thomson JA, Kim EE, Griffith JP, Navia MA, Murcko MA, Chambers SP, Aldape RA, Raybuck SA and Livingston DJ (1994) Structure and mechanism of interleukin-1 $\beta$ converting enzyme. Nature 370: 270-275

Yang X, Chang HY and Baltimore D (1998) Autoproteolytic activation of procaspases by oligomerization. Mol. Cell. 1: 319-325 